\title{
A melanin-mediated cancer immunotherapy patch
}

\author{
Yanqi Ye, ${ }^{1,2 *}$ Chao Wang, ${ }^{1,2 *}$ Xudong Zhang, ${ }^{1,2}$ Quanyin Hu, ${ }^{1,2}$ Yuqi Zhang, ${ }^{1,2}$ Qi Liu, ${ }^{1,2}$ Di Wen, ${ }^{1,2}$ \\ Joshua Milligan, ${ }^{1}$ Adriano Bellotti, ${ }^{1,3}$ Leaf Huang, ${ }^{1,2}$ Gianpietro Dotti, ${ }^{4}$ Zhen Gu ${ }^{1,2,3 \dagger}$
}

\begin{abstract}
Melanin is capable of transforming $99.9 \%$ of the absorbed sunlight energy into heat, reducing the risk of skin cancer. We here develop a melanin-mediated cancer immunotherapy strategy through a transdermal microneedle patch. B16F10 whole tumor lysate containing melanin is loaded into polymeric microneedles that allow sustained release of the lysate upon insertion into the skin. In combination with the near-infrared light irradiation, melanin in the patch mediates the generation of heat, which further promotes tumor-antigen uptake by dendritic cells, and leads to enhanced antitumor vaccination. We found that the spatiotemporal photoresponsive immunotherapy increases infiltration of polarized $T$ cells and local cytokine release. These immunological effects increase the survival of mice after tumor challenge and elicited antitumor effects toward established primary tumor and distant tumor. Collectively, melanin generates local heat, boosts T cell activities by transdermal vaccines, and promotes antitumor immune responses.
\end{abstract}

\section{INTRODUCTION}

Emerging technologies associated with immunotherapy hold tremendous promise in cancer therapy (1). Micro- or nanoformulations or engineered immune cells can be used to deliver a variety of immunomodulators $(2,3)$. Scaffolds, such as hydrogel, have also been developed to generate an immunogenic microenvironment that recruits and activates immune cells in situ (4-7). Moreover, T cell engineering that incorporates antibodies or therapeutics facilitates immune targeting and treatment (8-11). Also, dendritic cell (DC)-based vaccination can effectively capture antigens to improve the effectiveness of the immune response and is a powerful tool for cancer therapy (12-16). However, engineering of DCs often involves complex and expensive ex vivo manipulation $(4,17)$. In addition, the limited lymph node-homing capability of ex vivo manipulated DCs is, at least in part, responsible for the limited anticancer efficacy (18). Vaccination with whole tumor antigens provides a broad source of tumor-associated antigens that elicit substantially enhanced immune responses compared with narrowly defined tumor antigens (19). Moreover, presenting a broad spectrum of immunogenic epitopes not only augments the immunity mediated by the DC antigen uptake and processing but also improves the direct activation of $\mathrm{CD} 4^{+} \mathrm{T}$ helper and $\mathrm{CD} 8^{+} \mathrm{cy}$ totoxic T lymphocytes $(7,20)$.

Here, we describe a B16F10 melanoma vaccine patch that targets antigen-presenting cells (APCs) directly via transdermal delivery of tumor lysates combined with melanin (Fig. 1A). This vaccine involves the encapsulation of inactive whole tumor lysate that is gradually released by an intradermal microneedle (MN) patch inserted into the skin. MNs facilitate the uptake and presentation of antigens by DCs and, in turn, promote immune activation through the extensive network of lymphatic vessels in the dermis (21-23). At the same time, the presence of melanin, the existing natural biological

\footnotetext{
1 Joint Department of Biomedical Engineering, University of North Carolina at Chapel Hill and North Carolina State University, Raleigh, NC 27695, USA. ${ }^{2}$ Division of Pharmacoengineering and Molecular Pharmaceutics, Eshelman School of Pharmacy, University of North Carolina at Chapel Hill, Chapel Hill, NC 27599, USA. ${ }^{3}$ Department of Medicine, University of North Carolina School of Medicine, Chapel Hill, NC 27599, USA. ${ }^{4}$ Department of Microbiology and Immunology, Lineberger Comprehensive Cancer Center, University of North Carolina at Chapel Hill, Chapel Hill, NC 27599, USA. *These authors contributed equally to this work. tCorresponding author. Email: zgu@email.unc.edu
}

pigment in the whole tumor lysate, allows the local release of heat via remotely controllable near-infrared (NIR) light emission. Local heat causes the release of inflammatory cytokines that attract immune cells, generation of immunogenic substrates such as extracellular heat shock proteins (HSPs), reactive oxygen species (ROS), antigen adjuvants, and some other danger signals that activate the immune system $(24,25)$. The mild increase in the local temperature of the interstitial tissues also contributes to the increased blood and lymphatic flow that facilitates the migration of APCs and T cells (24) and finally initiates B16F10-specific immune responses $(23,26)$. The increased blood flow may also allow the recruitment of other cell subsets such as natural killer (NK) cells $(7,27,28)$. We found that the administration of the vaccine $\mathrm{MN}$ patch generated robust innate and adaptive immune responses and induced tumor regression in the B16F10 melanoma model. Moreover, the NIR-enhanced transdermal vaccination delayed the growth of distant tumor and improved long-term survival, paving a strong rationale for pursuing this strategy in clinical studies.

\section{RESULTS \\ Preparation and characterization of the cancer immunotherapy patch}

We first investigated whether the tumor lysate can be loaded and released from transdermal $\mathrm{MN}$ patches in a sustained manner. We fabricated the $\mathrm{MN}$ patch within a micromold to form hyaluronic acid-based MNs that encapsulate the whole tumor lysate (with melanin) and adjuvants, such as granulocyte-macrophage colonystimulating factor (GM-CSF) $(29,30)$. Patches with and without tumor lysate were illustrated from the axial and transverse perspectives, respectively (Fig. 1B). An array of $15 \times 15 \mathrm{MNs}$ was assembled on a $9 \mathrm{~mm}$ by $9 \mathrm{~mm}$ patch with a center-to-center interval of $600 \mu \mathrm{m}$. The detailed dimensions of the MNs were visualized by the scanning electron microscopy (Fig. 1C). Each MN had a conical construction with a diameter of $300 \mu \mathrm{m}$ at the base, a height of $800 \mu \mathrm{m}$, and a sharp tip tapering to a 5- $\mu \mathrm{m}$ radius of curvature. Upon loading with the tumor lysate, MNs appeared notably darker than the blank hyaluronic acid-based MNs because of the presence of melanin in the patch $(31,32)$. The amount of melanin was around $50 \mu \mathrm{g}$ per patch, which is within the safe dosage range of a single administration (33). A 

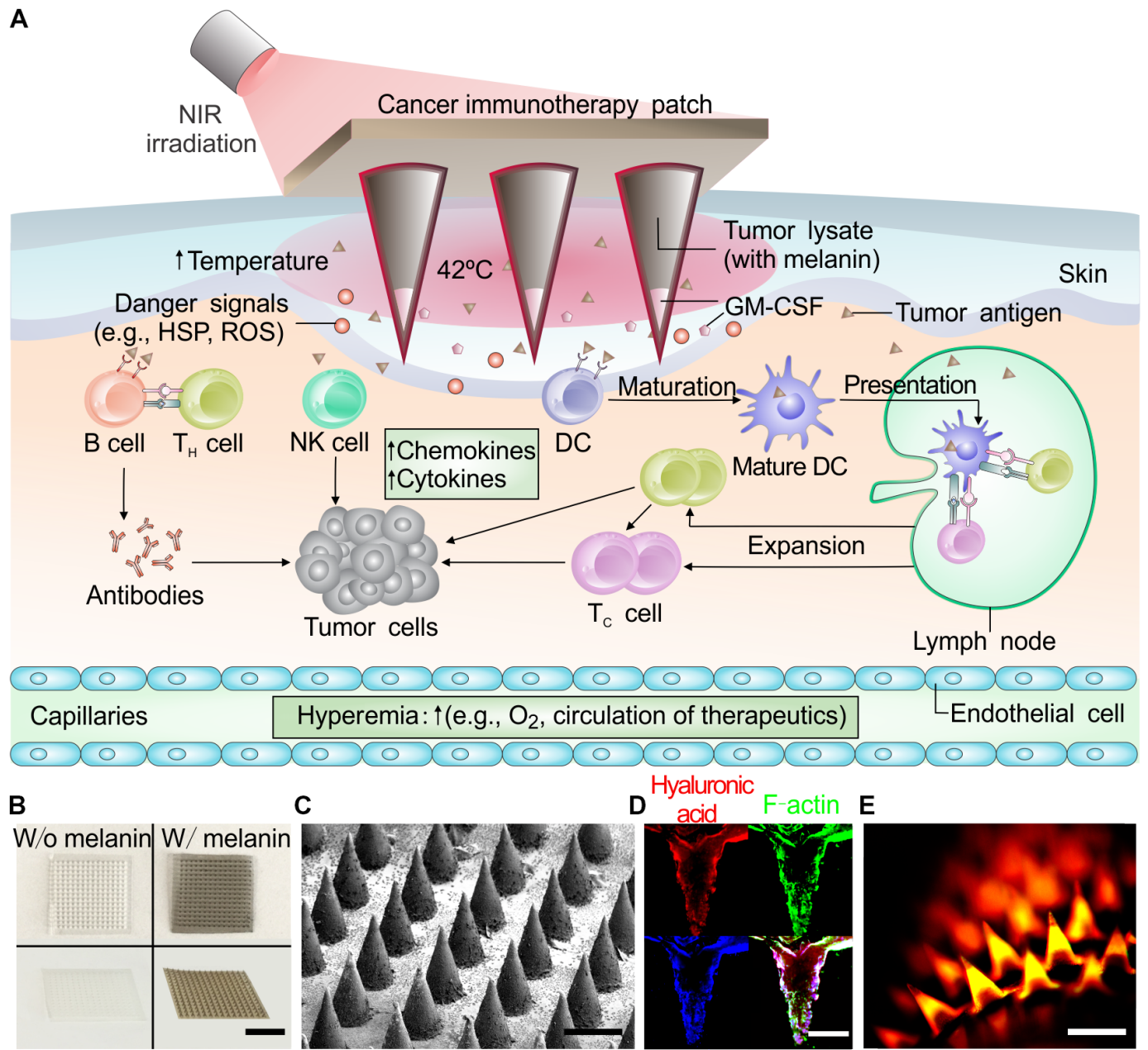

C

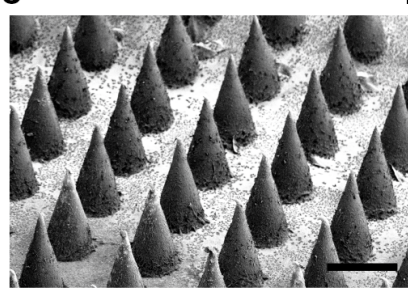

Hyaluronic

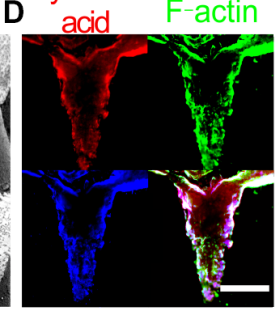

DNA Merged

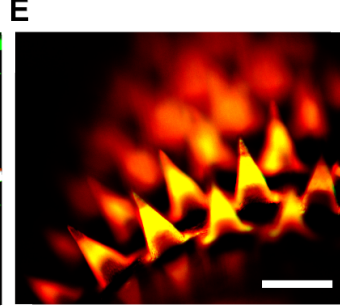

Fig. 1. Schematic of melanin-mediated cancer immunotherapy through a transdermal MN-based vaccine patch. (A) Schematic illustration of $M N$-based transdermal vaccination. $T_{H}$ cell, $T$ helper cell; $T_{c}$ cell, cytotoxic T cell. (B) Photograph of representative MN patches without (W/o) melanin and with (W/) melanin (scale bar, 4 mm). (C) Scanning electron microscopy image of the MN patch (scale bar, $400 \mu \mathrm{m}$ ). (D) Fluorescence cross-sectional images of a representative MN. Actin filaments in cells were visualized by Alexa Fluor 488 phalloidin (green), cell DNA fragments were stained with Hoechst (blue), and hyaluronic acid polymer matrix was labeled with rhodamine B (red) (scale bar, $200 \mu \mathrm{m}$ ). (E) Fluorescence imaging of a representative MN patch that contained the Alexa Fluor 488 phalloidin-labeled tumor lysate and rhodamine B-labeled hyaluronic acid (scale bar, $400 \mu \mathrm{m}$ ).

representative $\mathrm{MN}$ was constructed with rhodamine B-labeled hyaluronic acid encapsulating tumor lysate with actin filaments and DNA stained with phalloidin and Hoechst, respectively (Fig. 1D). A fluorescent view of the array further showed the uniform loading of tumor lysate and alignment of the MNs (Fig. 1E). The MN underwent failure at $0.39 \mathrm{~N}$ in strain testing while being compressed to a quarter of its height (fig. S1), demonstrating sufficient strength for potential skin insertion without buckling (34).

Next, we evaluated the effects of the NIR laser irradiation on melanin-induced heat generation. The temperature variations of the MNs were recorded in real time using an infrared thermal camera (Fig. 2A). Compared with the transparent hyaluronic acid-based MNs, the surface temperature of the MNs loaded with tumor lysate increased dramatically within 1 min because of the melanin-induced light-to-heat transduction (Fig. 2B). NIR irradiation at $808 \mathrm{~nm}$ could be absorbed by the melanin contained in the tumor lysate as demonstrated by the melanin's absorption spectrum (fig. S2A). The ab- sorption coefficient of the melanin content in the tumor lysate was also comparable with that of the synthetic melanin (fig. S2A and table S1). Despite the temperature increase, the morphology of the MNs remained unchanged, and the photothermal property of melanin was stable during the treatment (fig. S2B) (35). The heating behavior of the MN patch was also maintained when repeated NIR light exposures were performed (fig. S3). Furthermore, the steady state of the patch's surface temperature was lysate concentrationand NIR light intensity-dependent, whereas the thickness of the MN backing had a minimum effect (fig. S4 and Fig. 2C). The surface temperature of the patch could be controlled under hyperthermia $\left(42^{\circ} \mathrm{C}\right)$ to minimize the potential thermal-induced denaturation of the tumor antigen and other biomolecules.

\section{In vitro activation of $D C s$ in response to vaccine $M N$}

GM-CSF that serves as a potent cytokine for DC recruitment and activation was physically encapsulated into MN tips by cross-linking 

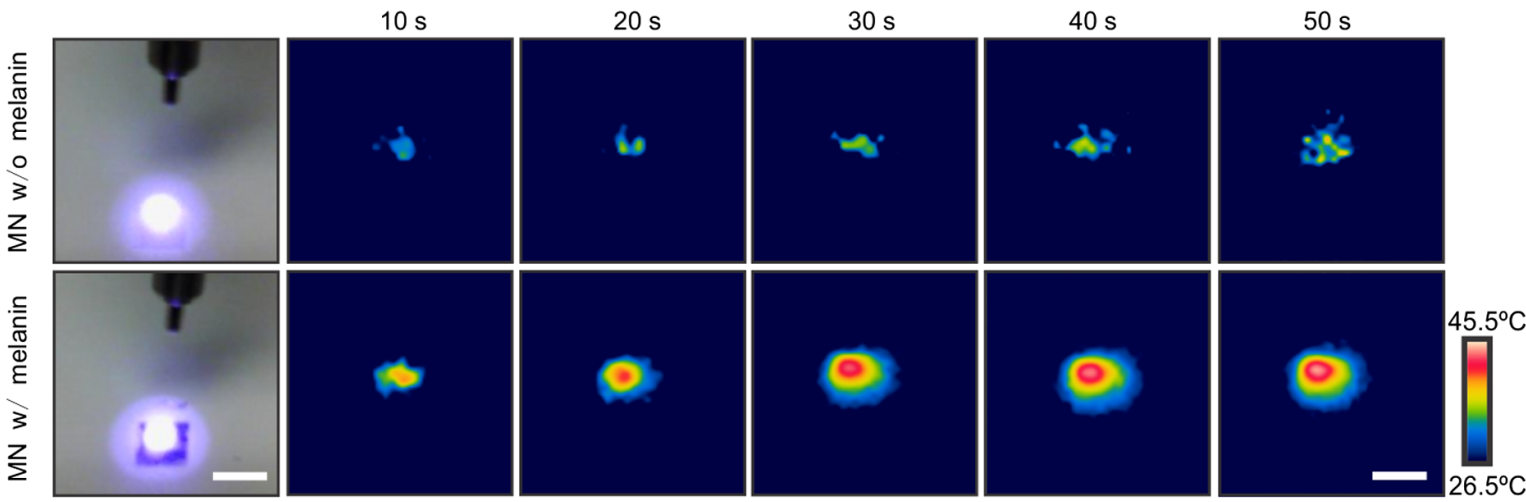

B

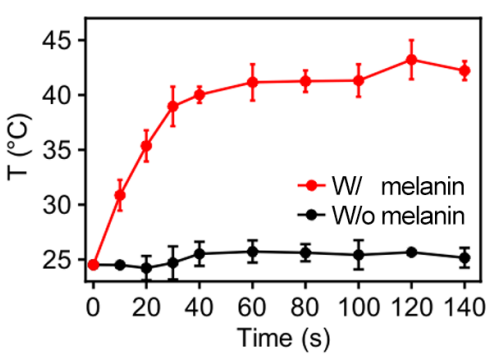

$\mathrm{E}$

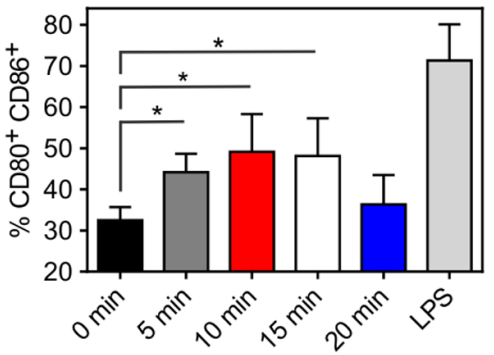

C

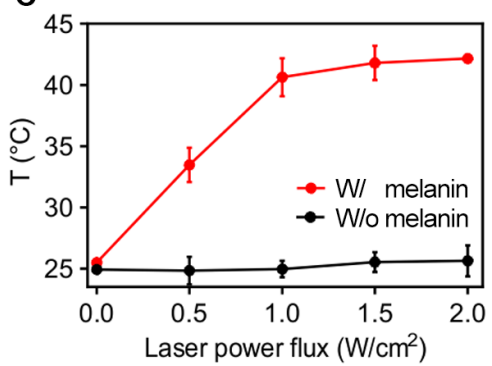

$\mathbf{F}$
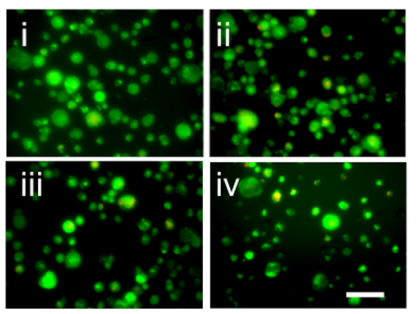

D

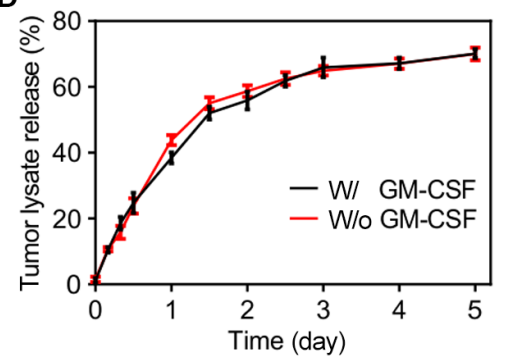

G

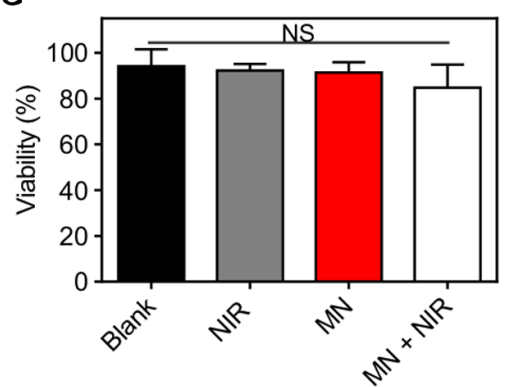

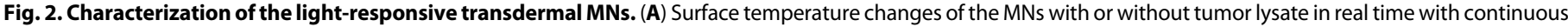

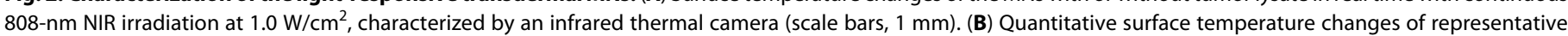

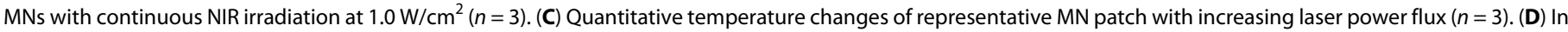

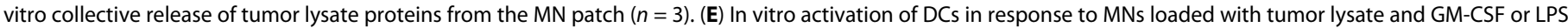

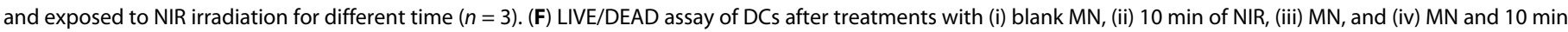

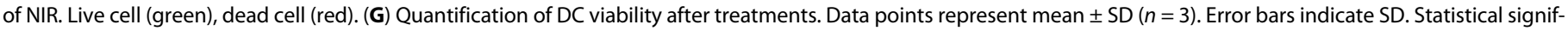
icance was calculated by Student's $t$ test [not significant (NS), $P>0.05$; ${ }^{*} P<0.05$ ].

methacrylated hyaluronic acid upon ultraviolet irradiation. We found that $60 \%$ of the bioactive GM-CSF was released from MN within 48 hours (fig. S5, A and B), whereas sustained release of the tumor lysate protein was observed over 5 days (Fig. 2D). Incorporation of the NIR treatment did not alter the release profiles of GM-CSF and tumor lysate (fig. S5, C and D). Scanning electron microscopy images of vaccine MNs showed a gradual dissociation of tips over time (fig. S6).

To evaluate whether GM-CSF in MNs provided signaling cues that could efficiently promote DC maturation, we exposed bone marrow-derived DCs to the dissolved MN suspension. The percentage of matured DCs $\left(\mathrm{CD}^{+} 0^{+}\right.$and $\left.\mathrm{CD}^{+} 6^{+}\right)$substantially increased from $36.7 \pm 2.3 \%$ to $48.9 \pm 3.1 \%$ after treatment with tumor lysate- and GM-CSF-loaded MNs with 10 min of NIR laser irradiation (Fig. 2E) (36). The effect of varying NIR laser irradiation time on the activation of DCs was also measured. Ten minutes of NIR irradiation allowed optimal DC activation as compared with samples treated with 5 or $15 \mathrm{~min}$ (Fig. 2E). Only $20 \mathrm{~min}$ of NIR exposure slightly impaired DC viability and functionality (fig. S7). DCs in all other experimental conditions of either MN suspension or $10 \mathrm{~min}$ of NIR irradiation exhibited high viability (Fig. 2, F and G, and fig. S8).

\section{In vivo efficacy of MN-mediated immunization upon NIR}

To characterize the in vivo efficacy of MN-based immunization, we transdermally treated C57BL/6J mice with vaccine $\mathrm{MN}$ patch loaded with B16F10 whole tumor lysate containing $1.5 \mathrm{mg}$ of extracted protein. We measured the magnitude and duration of the immune response after NIR irradiation and subsequent tumor challenge (Fig. 3A). $\mathrm{MN}$ patches were applied on the mice skin of the caudal-dorsal area for about $10 \mathrm{~min}$ and further affixed using the Skin Affix surgical 
A

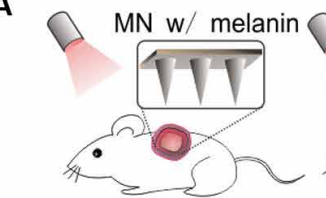

Light-to-heat conversion

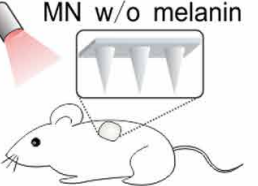

Limited heating

B

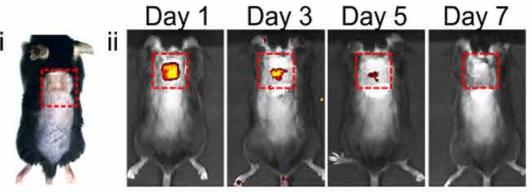

Fluorescence Radiance $\left(\mathrm{p} / \mathrm{s} / \mathrm{cm}^{2} / \mathrm{sr}\right)$

$\operatorname{Min} \square \operatorname{Max} \operatorname{Min}=1.13 \times 10^{8}, \operatorname{Max}=2.90 \times 10^{8}$

C

Blank
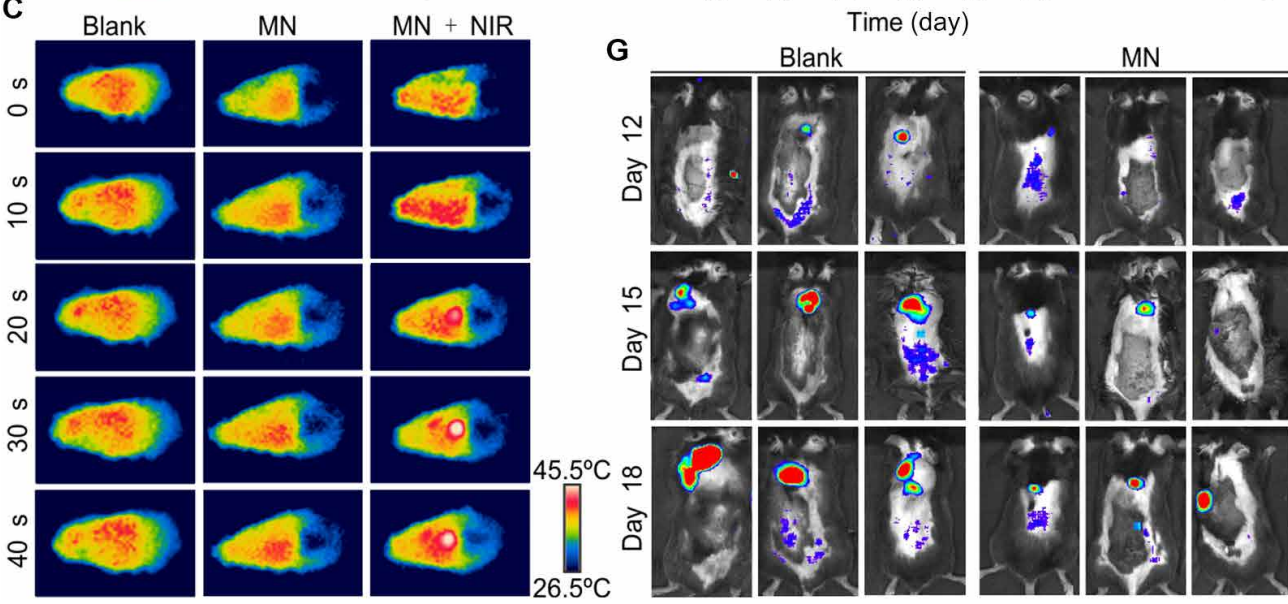

F
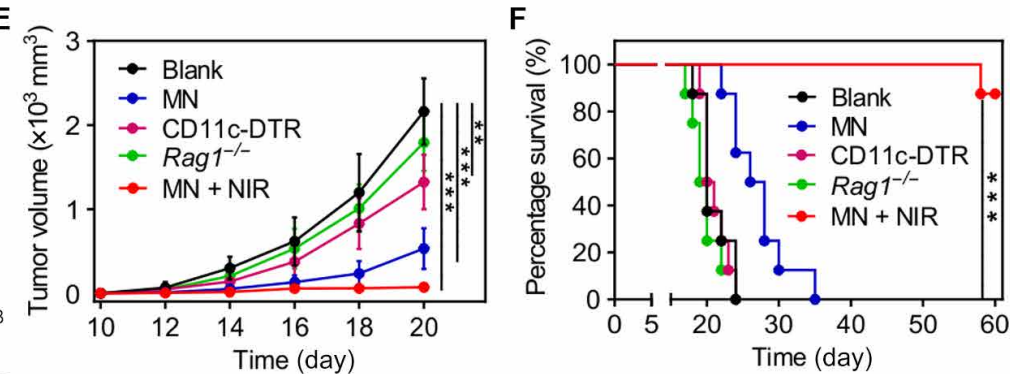

$\mathrm{MN}+\mathrm{NIR}$

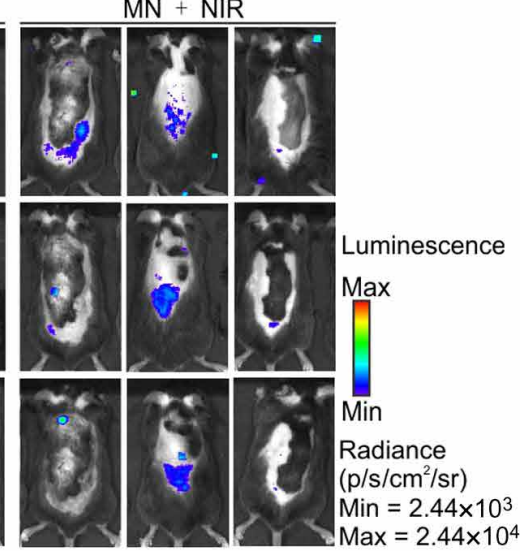

Fig. 3. Vaccine MN confer protective innate and adaptive immunity. (A) Schematic illustration of MN cancer immunotherapy. (B) Characterization of the MNs after insertion. (i) Photo of mouse dorsal skin (the area within the red line) that was treated transdermally with one MN patch and (ii) fluorescence signals of Cy5.5-labeled MN patch over time. (C) Surface temperature changes of individual animal after MN insertion into the skin measured by an infrared thermal camera. (D) Schematic representation of the B16F10 vaccine tumor model. (E) Average tumor volumes in treated mice after tumor challenge. (F) Kaplan-Meier survival curves for treated and control mice. Data points represent mean \pm SD $(n=8)$. Error bars indicate SD. Statistical significance was calculated by Student's test and log-rank test $\left.{ }^{* *} P<0.01 ;{ }^{* * *} P<0.001\right)$. (G) In vivo bioluminescence imaging of the B16F10 melanoma in different experimental groups and at different time points after tumor challenge. Three representative mice per treatment group are shown. The tumor growth in $(E)$ to $(G)$ was measured 10 days after tumor cell inoculation.

adhesive. Staining with trypan blue and hematoxylin and eosin (H\&E) indicated successful penetration of MNs in the excised skin (fig. S9). The transdermal patch remained in the skin for at least 5 days (Fig. 3B). We then performed localized NIR irradiation on the $\mathrm{MN}$ region for 10 min daily for 5 days (MN + NIR). Control mice were treated with either vaccine MN patch without NIR irradiation (MN), MN patch loaded only with melanin (melanin), or MNs containing only hyaluronic acid with NIR irradiation (blank).

Temperature changes in the regional skin surface after vaccine $\mathrm{MN}$ insertion were recorded in real time using an infrared thermal camera. Light-to-heat transduction upon NIR irradiation caused a local heating effect observed in mice treated with vaccine MNs (Fig. 3C). The melanin in the vaccine patch mediated the transdermal heating at temperatures between $38^{\circ}$ and $42^{\circ} \mathrm{C}$ within $30 \mathrm{~s}$. Mild hyperthermia at the local treated site was similarly observed in mice treated with synthetic melanin-loaded MNs (fig. S10A). In contrast, mice treated with blank MNs and NIR and mice implanted with loaded MNs but without NIR showed limited variations in skin surface temperature within the normal range of $33^{\circ}$ to $36^{\circ} \mathrm{C}$ (Fig. 3C).

In a prophylactic mouse model, mice were implanted with B16F10 melanoma cells 10 days after vaccination (Fig. 3D). All mice treated with the blank MNs had appreciable tumor growth within 15 days after tumor cell inoculation and required euthanasia by day 25 . MNs loaded with melanin and treated with NIR irradiation slightly improved the survival of the mice because some mice survived to day 25 (fig. S10, B and C). Similarly, MNs loaded with tumor lysate and melanin but without NIR irradiation caused tumor protection in $13 \%$ of the mice until day 30 (Fig. 3, E and F). In sharp contrast, mice receiving the combined vaccination (MNs loaded with tumor lysate and GM-CSF and NIR irradiation) showed long-term survival with complete tumor rejection in $87 \%$ of the treated mice (Fig. 3, E and $\mathrm{F}$, and fig. S11). Bioluminescence imaging of the B16F10 melanomabearing mice confirmed significant inhibition of tumor growth (Fig. 3G and fig. S12). This was further evidenced by the measurement of tumor weight (fig. S13) and histologic analysis (fig. S14A).

We next assessed the requirement of immune cells for the antitumor effects observed by combined vaccination-based treatments. Depletion of CD11 $\mathrm{c}^{+}$DCs in diphtheria toxin receptor (DTR) mice was sufficient to abrogate the antitumor effect of the vaccine MN (Fig. 3, E and F). A study in Rag1 ${ }^{-/-}$mice deficient in T cells and B cells showed a significant loss of tumor growth suppression during treatment with combined vaccination (fig. S11). Selective depletions of $\mathrm{CD}^{+}$and $\mathrm{CD}^{+}{ }^{+} \mathrm{T}$ cells before combined $\mathrm{MN}$ vaccination were also studied. Eliminating $\mathrm{CD} 8^{+} \mathrm{T}$ lymphocytes showed no significant 
tumor regression compared with blank control (fig. S15). When antiCD4 antibody was given to the mice, there was a decrease $(P<0.01)$ in tumor size in contrast to the control, suggesting the benefit of CD4 $\mathrm{T}$ cells to a lesser extent than CD8 $\mathrm{T}$ cells for the antitumor response (fig. S15). The depletion of B cells and NK cells also had deleterious impact on the immune response while not diminishing the vaccination effect toward tumor challenge (fig. S15). Together, these results showed that the $\mathrm{MN}$ vaccination was associated with $\mathrm{CD} 11 \mathrm{c}^{+}$ DCs and other immune cells such as T cells, B cells, and NK cells.

Loading of GM-CSF in the MNs played an important role in the local recruitment of DCs [CD $11 \mathrm{c}^{+}$, paired immunoglobulin (Ig)-like receptors (PIRs) of activating-A/B ${ }^{+}$] (Fig. 4, A and C). Three days after combined vaccination, a 5.9-fold increase in accumulated DCs was observed in the skin section compared with mice treated with blank MN. NIR further augmented the effect of GM-CSF-loaded MNs on recruiting DCs (Fig. 4C). Increased localization of NK cells was also observed (Fig. 4, B and D). In addition, the elevated local microcirculatory blood perfusion observed after NIR and MN treatment could contribute in enhancing the migration of immune cells (table S2 and fig. S16). Tumor infiltration by T cells upon treatment was analyzed by flow cytometry on day 15 after tumor inoculation. About 9.8-fold increase in $\mathrm{CD}^{+} \mathrm{T}$ cells was observed in mice receiving the combined vaccination compared with control mice, whereas $\mathrm{MN}$-only group showed a 5.8-fold increase (Fig. 5A). Furthermore, staining with $\mathrm{H}-2 \mathrm{D}^{\mathrm{b}}$ gp100 tetramers identified B16F10specific $\mathrm{CD} 8^{+} \mathrm{T}$ cells in tumors of treated mice (37). The percentage of tetramer-positive $\mathrm{CD}^{+} \mathrm{T}$ cells was found to be greater in the $\mathrm{MN}$-treated mice compared with control mice lacking immunization (fig. S17). Mice with combined treatment or MN-only treatment exhibited 1.5- and 1.3-fold increases in activated DCs $\left(\mathrm{CD}^{+} 0^{+}\right.$and $\mathrm{CD}^{+} 6^{+}$), respectively, in regional skin as compared with control mice (Fig. 5B). Immunofluorescence staining and in situ cell apoptosis confirmed the results obtained with flow cytometry (Fig. 5C and fig. S14B). Local immune activation was associated with systemic immune responses. We measured an eightfold increase in IgG titers in the serum of immunized mice as compared with mice treated with blank control (Fig. 5D). NIR treatment promoted further increase in IgG titers by day 15 and prolonged immune responses compared with
A

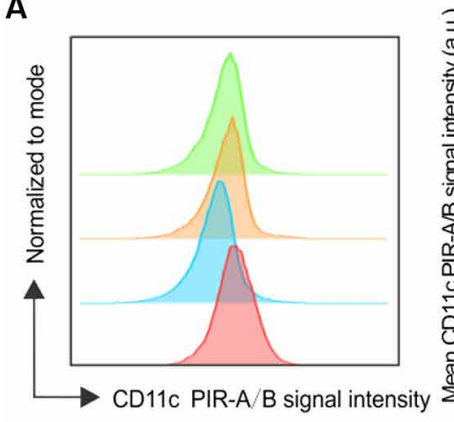

C

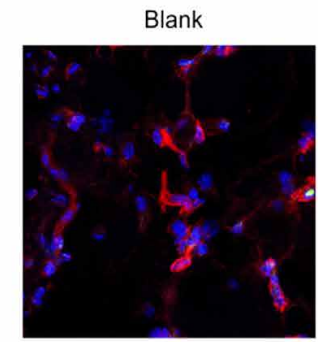

D

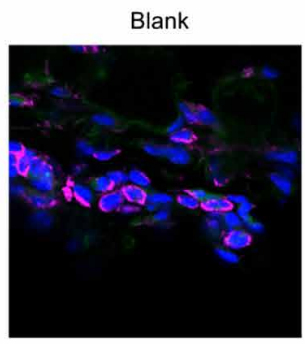

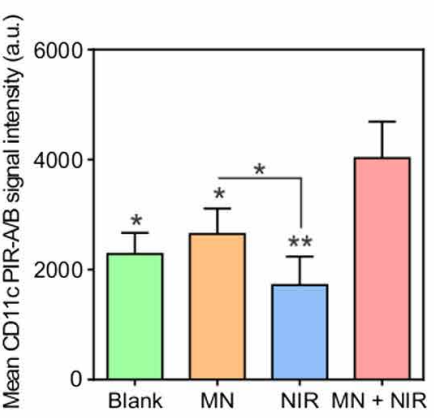

$\mathrm{MN}$
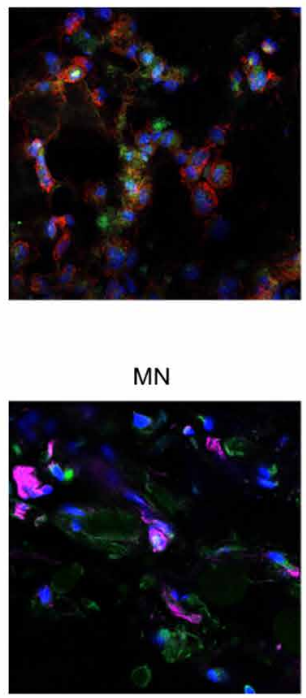
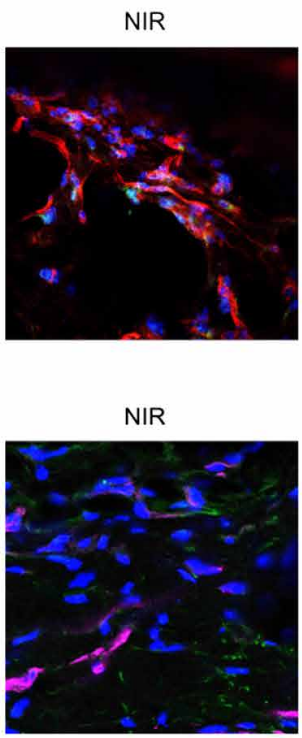

B

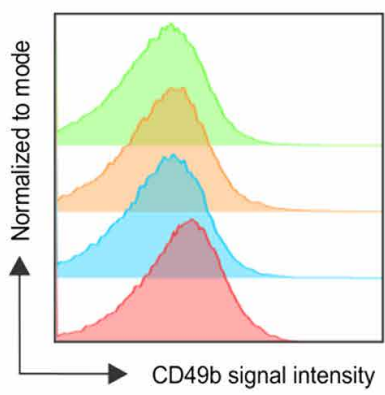

$M N+N I R$

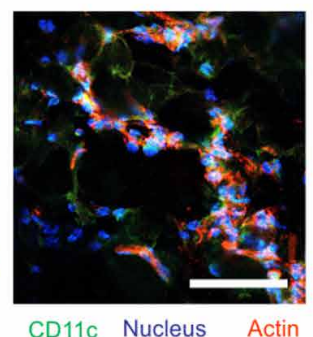

$\mathrm{MN}+\mathrm{NIR}$

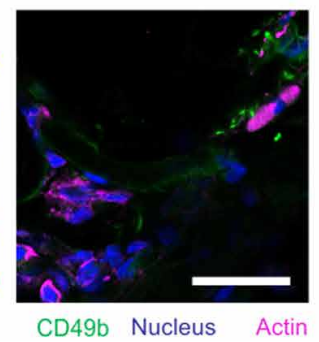

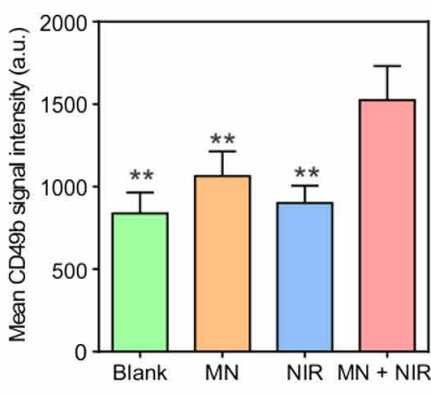
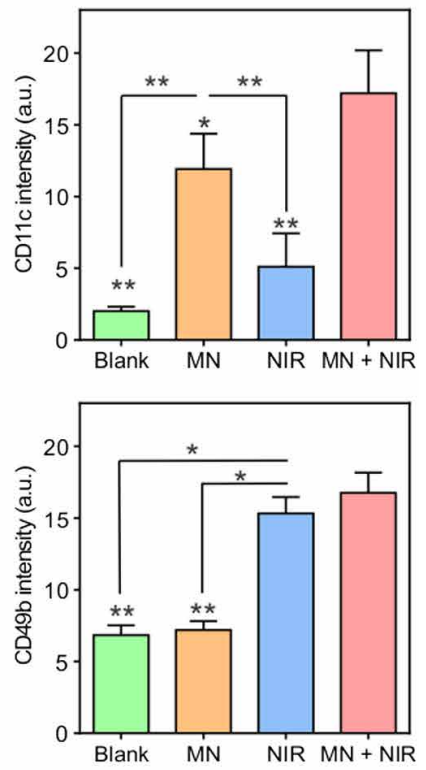

Fig. 4. Immune cell recruitment after the NIR-boosted and MN-mediated cancer immunotherapy. (A) Representative quantitative analysis of DCs (CD11 $\mathrm{C}^{+}$and PIR-A/B ${ }^{+}$) infiltrated in the skin 3 days after treatments as assessed by flow cytometry. The indicated samples were treated with blank MN (blank), vaccine MN (MN), MN loaded with tumor lysate without GM-CSF and treated with NIR (NIR), and vaccine MN and treated with NIR (MN + NIR). a.u., arbitrary units. (B) Representative quantitative analysis of NK cells (CD49b ${ }^{+}$) in the skin upon transdermal cancer immunotherapy as assessed by flow cytometry. (C and $\left.\mathbf{D}\right)$ Immunofluorescence staining and quantitative analysis of $(C) C D 11 c^{+} D C s$ (scale bar, $100 \mu \mathrm{m}$ ) and (D) CD49b ${ }^{+}$NK cells (scale bar, $100 \mu \mathrm{m}$ ). Statistical significance was calculated by Student's $t$ test $\left({ }^{*} P<0.05 ;{ }^{*} P<0.01\right)$. Asterisks indicate significant differences between the MN + NIR group and all other treatment groups. Data points represent mean \pm SD $(n=3)$. Error bars indicate SD. 
A

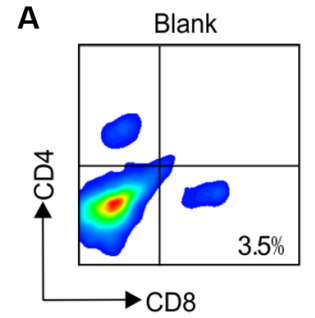

B Blank

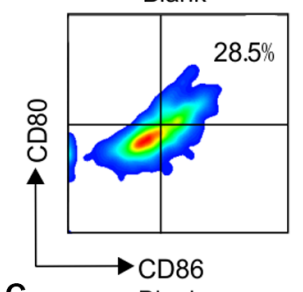

C

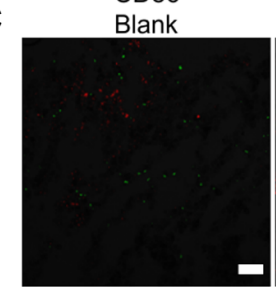

$\mathrm{F}$

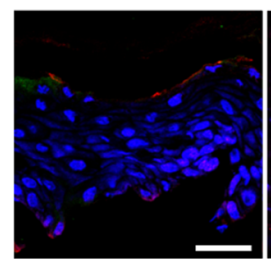

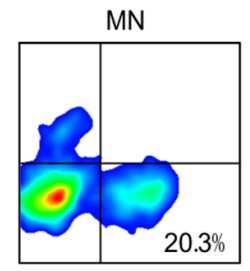

MN

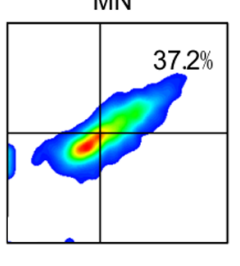

MN
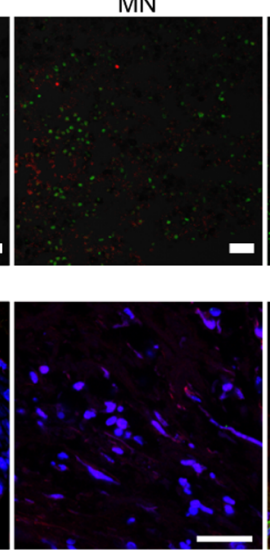

$\mathrm{MN}+\mathrm{NIR}$

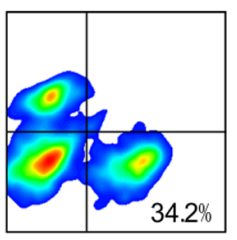

$\mathrm{MN}+\mathrm{NIR}$

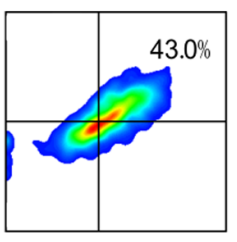

$\mathrm{MN}+\mathrm{NIR}$

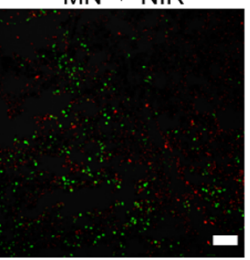

$\mathrm{CD}^{+} \mathrm{CD}^{+}$

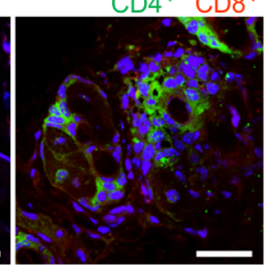

HSP70 Nucleus Actin

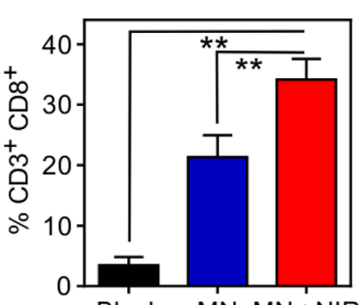

Blank MN MN+NIR

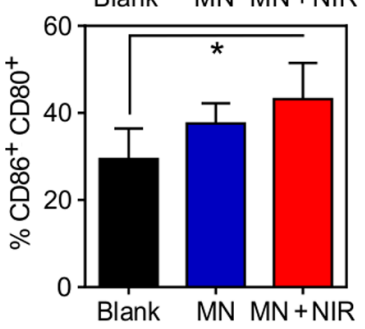

G

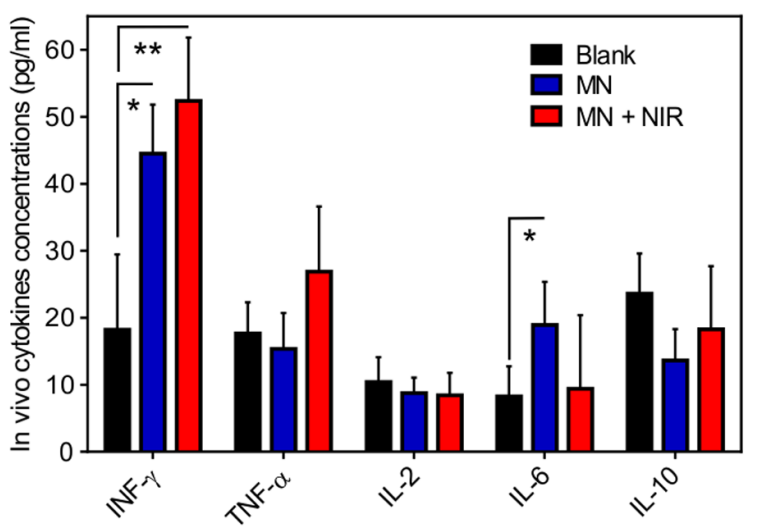

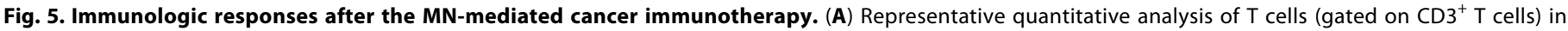

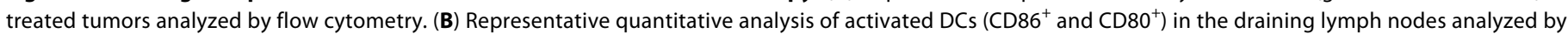

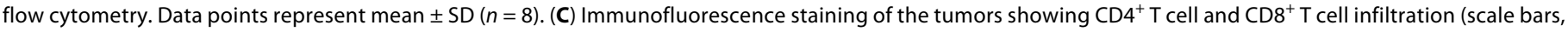

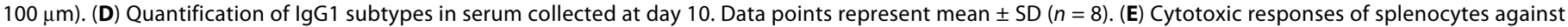

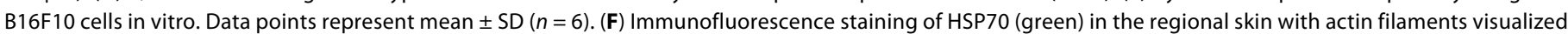

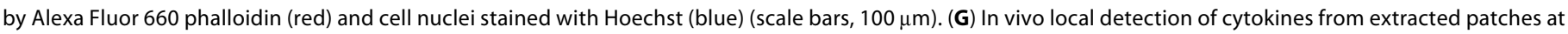

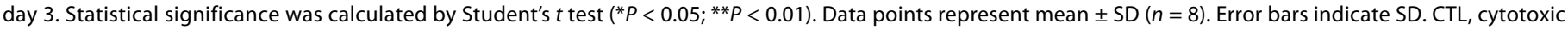
T lypmphocyte.

control groups (fig. S18). The in vitro analysis of splenocytes revealed 10-fold higher frequency of $\mathrm{T}$ cells responding to B16F10 tumor lysate in mice receiving the combined vaccination (Fig. 5E).

To examine that the light-to-heat transduction induced the production of danger signals and proinflammatory cytokines, we first measured local levels of ROS in the MN-treated surrounding tissue by flow cytometry. Samples from mice receiving the combined treatment showed about fourfold increase in ROS levels compared with the untreated group, whereas 1.5-fold increase was observed compared with the MN control without the NIR irradiation (fig. S19). In line with the elicitation of danger signals, combined vaccination caused the expression of HSP70 and HSP90 (Fig. 5F and fig. S20) (38). Danger signals from the local tissue and the antigenic molecules promote proinflammatory cytokine production (39). Consistent with this effect, we found local increases of interferon- $\gamma$ (IFN- $\gamma$ ), tumor necrosis factor- $\alpha$ (TNF- $\alpha$ ), and interleukin-6 (IL-6) in mice treated with the combined vaccination compared with MN controls (Fig. 5G and fig. S21).

\section{Efficacy of MN patch in distant tumors}

We further analyzed whether the local vaccination confers protection toward secondary tumors distant from the NIR- and MN-treated site (Fig. 6A). Local NIR irradiation and MN treatment were performed only on the left-side tumor in B16F10 mice bearing bilateral tumors. Right-side tumor was not injected and was shielded from light (Fig. 6B). We observed that the tumor sizes and bioluminescence signals decreased significantly on both sides of the mice that had the combined vaccination (Fig. 6, C to G). Meanwhile, substantial increase of activated DCs in the regional lymph node (Fig. 5B) and enhanced cytotoxic responses to B16F10 cells of the splenocytes in vitro (Fig. 5E) indicated that systemic antitumor effect could be achieved by the transdermal immunotherapy. This effect was parallel by fivefold increases in $\mathrm{CD}^{+} \mathrm{T}$ cell infiltration compared with control, which was consistent with the role of immune cells for antitumor efficacy (Fig. 6D). Distant metastases were not observed in the lungs of the mice with combinational treatment (fig. S22). 


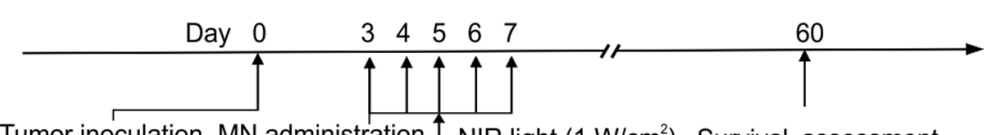

Tumor inoculation $\mathrm{MN}$ administration $\iota_{\text {NIR }}$ light $\left(1 \mathrm{~W} / \mathrm{cm}^{2}\right)$ Survival assessment

C

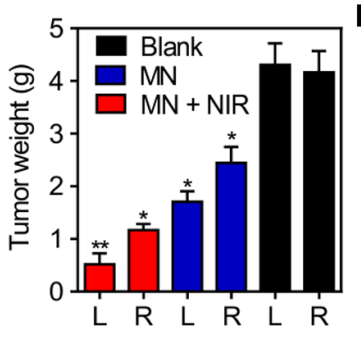

D

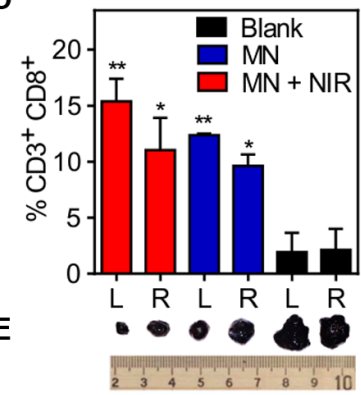

I

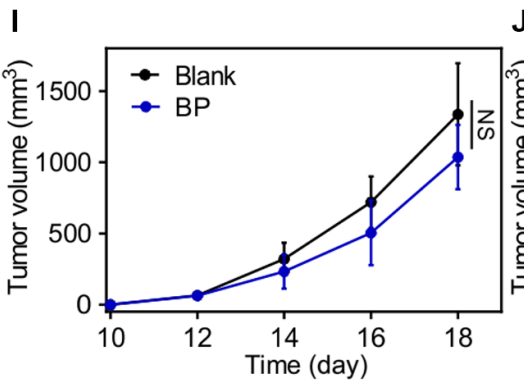

$F$

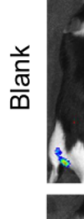

$\Sigma$
Day 7

Day 8

Day 9

Day 12

4 k.
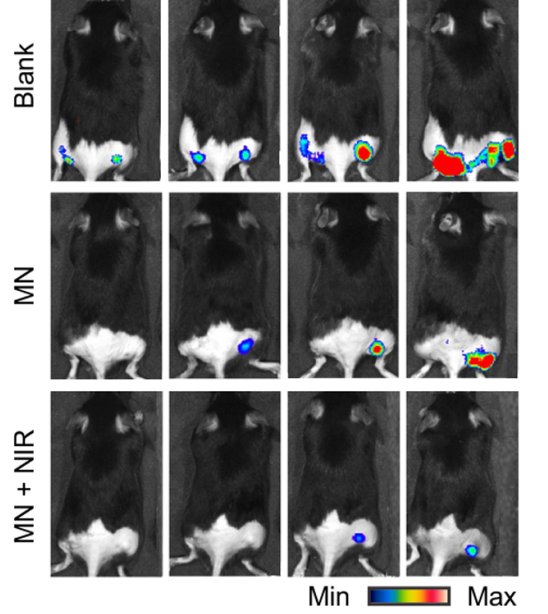

Luminescence Radiance $\left(\mathrm{p} / \mathrm{s} / \mathrm{cm}^{2} / \mathrm{sr}\right)$ $\operatorname{Min}=2.44 \times 10^{3}, \operatorname{Max}=2.44 \times 10^{4}$

J

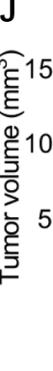

G
B
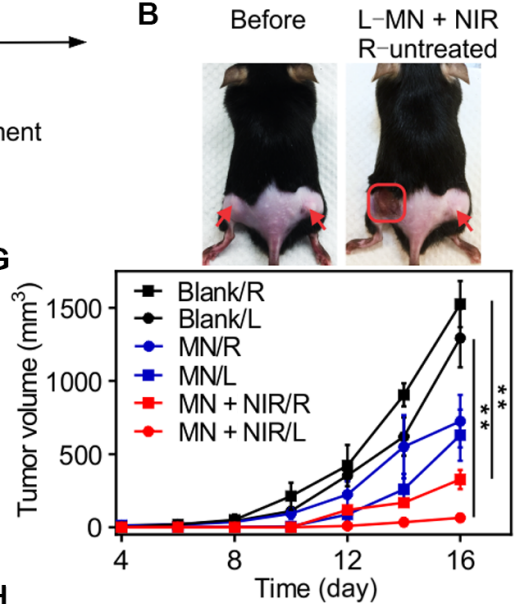

H

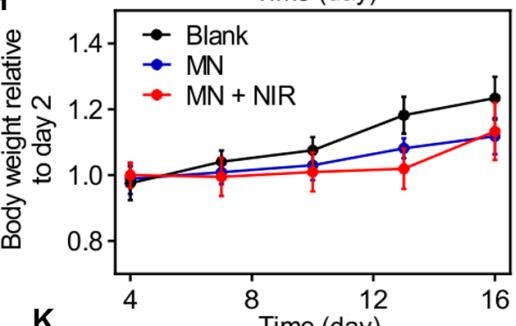

$\mathrm{K}$

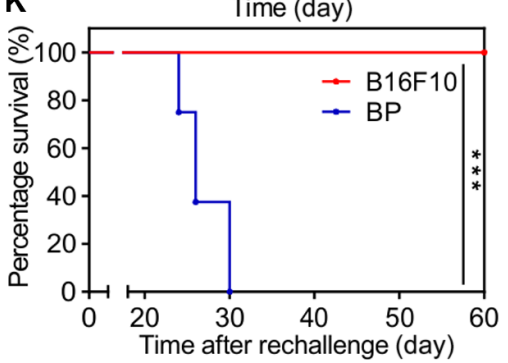

Fig. 6. Antitumor effect of local cancer immunotherapy treatment toward distant B16F10 tumors. (A) Schematic representation of the B16F10 tumor model. (B) Photograph of a mouse before and after MN administration. Red arrows indicate established tumors on both sides. $L$, left; $R$, right.The red line indicates the $M N$ injection site. (C) Tumor weights in different experimental groups $(n=3)$. (D) Tumor-infiltrating CD8 ${ }^{+}$T cells after treatments in different experimental groups $(n=6)$. Asterisks in (C) and (D) indicate statistically significant differences between blank MN and other groups. (E) Images of tumors extracted from treated mice indicated by the labels in (D). (F) In vivo bioluminescence imaging of treated B16F10 melanoma at different time points after treatment. One representative mouse per treatment group is shown. (G) Average tumor volumes in treated mice. (H) Body weights of treated mice $(n=6)$. (I) Average B16F10 tumor volumes in mice treated with MNs loaded with BP lysate with melanin and blank MN $(n=8)$. (J) Average tumor volumes in vaccinated mice rechallenged with either B16F10 cells or BP cells on day 80 . (K) Kaplan-Meier survival curves for rechallenged mice. Data points represent mean \pm SD $(n=8)$. Error bars indicate SD. Statistical significance was calculated by Student's $t$ test and log-rank test (NS, $\left.P>0.05 ;{ }^{*} P<0.05 ;{ }^{* *} P<0.01 ; * * P<0.001\right)$.

Body weight measurements indicated that the treated mice gained weight within the normal ranges (Fig. $6 \mathrm{H}$ ). When the mice were vaccinated with tumor lysate of a different melanoma cell type $\left[B R A F^{V 600 E} P T E N^{-/-}\right.$Duke-clone 6 cell line (BPD6 or BP), syngeneic with C57BL/6J] $(40,41)$, minimal changes in the B16F10 tumor growth were observed (Fig. 6I), indicating the specificity of the immunological memory. Similarly, when vaccinated mice were rechallenged with B16F10 cells or BP cells, tumor protection was observed only in mice challenged with B16F10 (Fig. 6, J and K, and fig. S23).

\section{Efficacy of MN patch in other tumor models}

To demonstrate that the potency of proposed vaccination is not limited to the B16 melanoma model in which there is an up-regulation of the melanogenesis, we used a $B R A F^{V 600 E}$-mutated $\mathrm{BP}$ melanoma in C57BL/6J mice and a triple-negative breast cancer $4 \mathrm{~T} 1$ carcinoma tumor in $\mathrm{BALB} / \mathrm{cJ}$ mice. Studies of MN loaded with synthetic melanin (with the same amount of pigment as quantified by spectrophotometry; table S1) and tumor lysate showed similar hyperthermic effect and enhanced immune responses with combined vaccination (fig. S24). Tumor regression and long-term survival were also achieved. Vaccination with the combined approach rendered 75 and $87 \%$ of mice resistant to BP and 4T1 engraftment, respectively (Fig. 7, A and $\mathrm{B}$, and fig. S25). In the tumor-bearing mice, $\mathrm{MN}$ and NIR treatments induced complete remissions in 87 and $37 \%$ of mice engrafted with $\mathrm{BP}$ and $4 \mathrm{~T} 1$ cells, respectively (Fig. 7, C and D, and fig. S25). Analysis of the local HSP70 expression showed a 2.5-fold increase in BP and a 4 -fold increase in $4 \mathrm{~T} 1$ models upon combined vaccination compared with the MN alone (fig. S26). Production of proinflammatory cytokines was also induced with the combined treatment, resulting in enhanced DC activation (figs. S27 and S28). Treatments were well 


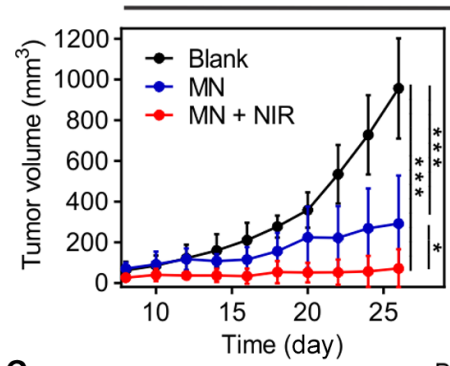

C

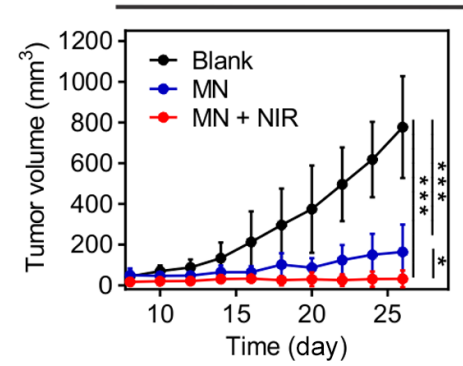

BP vaccine

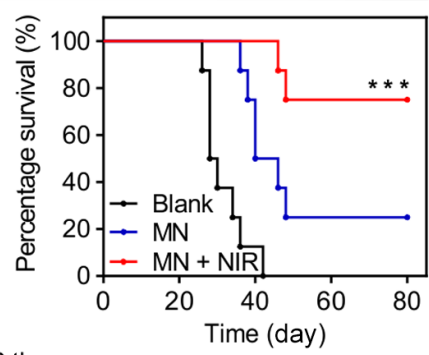

BP therapy

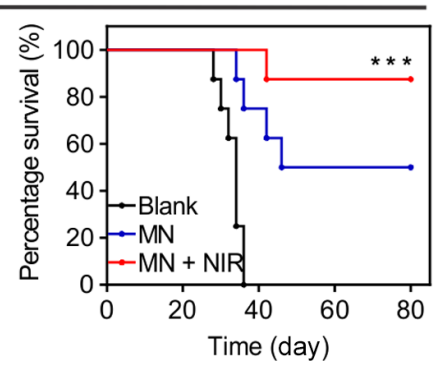

B

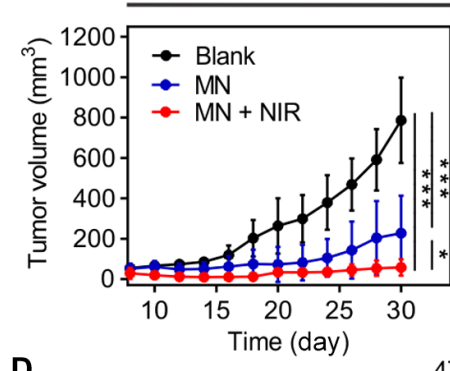

D

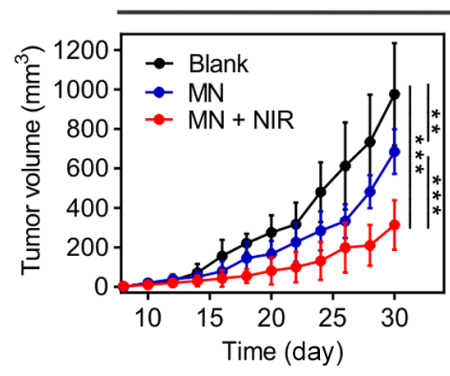

4T1 vaccine

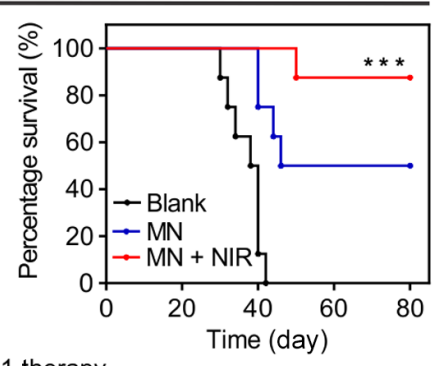

TT1 therapy

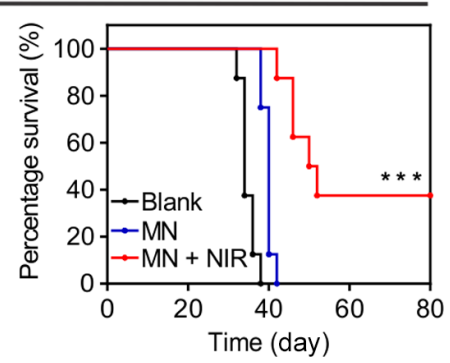

Fig. 7. Antitumor effect of local cancer immunotherapy treatment in various tumor models. (A) Average tumor growth and Kaplan-Meier survival rate of vaccinated C57BL/6J mice after BP tumor cell challenge. Mice were pretreated with blank MN (blank), MN loaded with BP tumor lysate and melanin (MN), or loaded MN combined with NIR irradiation (MN + NIR). (B) Average tumor growth and Kaplan-Meier survival rate of vaccinated BALB/cJ mice after 4T1 tumor cell challenge. Mice were pretreated with blank MN, MN loaded with 4T1 tumor lysate and melanin, or loaded MN combined with NIR irradiation. (C) Average tumor growth and Kaplan-Meier survival rate of C57BL/6J mice bearing established BP tumors. Mice were treated with blank MN, MN loaded with BP tumor lysate and melanin, or loaded MN combined with NIR irradiation. (D) Average tumor growth and Kaplan-Meier survival rate of BALB/cJ mice bearing established 4T1 tumors. Mice were treated with blank MN, MN loaded with 4T1 tumor lysate and melanin, or loaded MN combined with NIR irradiation. Data points represent mean \pm SD $(n=8)$. Error bars indicate SD. Statistical significance was calculated by Student's $t$ test and log-rank test $\left({ }^{*} P<0.05 ;{ }^{* *} P<0.01 ;{ }^{* * *} P<0.001\right)$.

tolerated and did not cause weight loss or clinical signs of distant metastasis (figs. S29 and S30).

\section{DISCUSSION}

In the current study, we have integrated the B16F10 whole tumor lysate and GM-CSF into a transdermal MN patch to sustain the delivery of the cancer vaccine and target immune cell populations in the epidermis. By incorporating the melanin, we have demonstrated that the combined vaccine facilitated local immune activation upon NIR irradiation through the recruitment of DCs and other immune cells. Compared with other photosensitizing agents $(42,43)$, melanin is a natural pigment with high biocompatibility and broad absorption spectrum. The efficient light-to-heat transduction mediated by melanin contributed to the rapid increase in skin temperature toward $42^{\circ} \mathrm{C}$.

The combined treatment caused long-term survival with tumor rejection in $87 \%$ of vaccinated C57BL/6J mice. Complete tumor protection was further observed in mice rechallenged with B16F10 tumor cells. After NIR treatment, the elevated local microcirculatory blood perfusion was associated with the migration of local DCs and NK cells. Depletion of B cells, NK cells, and T cells attenuated the treatment efficacy in vaccinated mice, highlighting the relevance of the immune control in the tumor development. In accordance, the production of danger signals and proinflammatory cytokines triggered the immune activation. We have identified the elevated HSP70 and HSP90 expression and ROS levels in the surrounding tissues. This effect was also associated with local enrichment of IFN- $\gamma$, TNF- $\alpha$, and IL- 6 . Characterization of other relevant danger signals and antigen adjuvants needs to be further examined.
In summary, the whole tumor lysate with melanin in a lightirradiated MN patch generates a hyperthermic-mimicking microenvironment that effectively recruits and activates immune cells at the vaccination site. As a result, the melanin-mediated patch prevents tumor engraftment in prophylactic models and causes sustained tumor regression in tumor-bearing mice. In addition, the natural melanin used in this treatment can also be extended to other biological pigments, such as carotenoid, xanthophylls, and bilirubin, for photomediated therapy. This method is also adaptable to deeptissue photoacoustic imaging, biological labeling, and targeting a variety of diseases in a photo-/thermoresponsive manner (44-46). Nevertheless, the parameters associated with this immune activation need further optimization, for instance, the duration of NIR irradiation and the selection of light wavelength, as well as the treatment site. For the future study of the proposed approach, personal neoantigens and checkpoint inhibitors could be incorporated into the MNs to further enhance treatment specificity and efficacy (47). For the potential translation of the vaccine patch, it relies profoundly on a prolonged safety evaluation of the local and systemic toxicity, as well as other potential side effects.

\section{MATERIALS AND METHODS Study design}

The objective of this study was to assess the effect of a melanin-mediated transdermal patch on cancer vaccine-based immunotherapy. The B16F10 whole tumor lysate containing melanin was integrated into an $\mathrm{MN}$ patch that allows controlled release of the cancer vaccine. 
Upon NIR light irradiation on the patch, the local heating effect on boosting immune responses was evaluated. Mice subjects were purchased from the Jackson Laboratory, weighed, and randomly divided into different experimental groups. The numbers of sampling and experimental replicates were included in each figure legend.

\section{Preparation and characterization of MNs}

All MNs were prepared using silicone molds with arrays of conical holes machined by laser ablation (Blueacre Technology Ltd.). Each MN had a $300 \mu \mathrm{m}$ by $300 \mu \mathrm{m}$ round base tapering to a height of $800 \mu \mathrm{m}$ with a tip radius of around $5 \mu \mathrm{m}$. MNs were arranged in a $15 \times 15$ array with a $600-\mu \mathrm{m}$ center-to-center spacing. GM-CSF solution $(0.1 \mathrm{mg} / \mathrm{ml}, 10-\mu \mathrm{l}$ final volume) was directly deposited by pipetting onto each silicone micromold surface, followed by vacuum $(600 \mathrm{mmHg})$ condition for $5 \mathrm{~min}$ to allow the solution to flow into the cavities. After that, $0.2 \mathrm{ml}$ of 4.0 weight $\%$ (wt \%) methacrylated hyaluronic acid solution mixed with $N, N^{\prime}$-methylenebisacrylamide (2.0 wt \%) and photoinitiator (Irgacure 2959; 0.05 wt \%) were directly deposited by pipetting onto each silicone micromold surface, followed by vacuum $(600 \mathrm{mmHg})$ condition for $5 \mathrm{~min}$ and centrifugation at $2000 \mathrm{rpm}$ for $5 \mathrm{~min}$ (Hettich Universal 32R centrifuge). GM-CSF layer was cross-linked via ultraviolet irradiation (wavelength, $365 \mathrm{~nm}$ ) for $10 \mathrm{~s}$, and a piece of $4 \mathrm{~cm}$ by $9 \mathrm{~cm}$ silver adhesive tape was applied around the $2 \mathrm{~cm}$ by $2 \mathrm{~cm}$ micromold baseplate. Three milliliters of homogenized B16F10 tumor lysate (containing $1.5 \mathrm{mg}$ of extracted tumor lysate proteins) in $4.0 \mathrm{wt} \%$ mixed methacrylated hyaluronic acid solution was added to the prepared micromold reservoir. We also used hyaluronic acid solution with different concentrations of tumor protein from 0.25 to 0.5 to $1.0 \mathrm{mg} / \mathrm{ml}$. Protein concentration was quantified by measuring extracted protein content in the tumor lysate supernatant using T-PER (tissue protein extraction reagent) and Bradford assay before the MN fabrication. For the BP or 4T1 tumor lysate-loaded MNs, 50- $\mu$ g melanin was dissolved in 4.0 wt \% mixed methacrylated hyaluronic acid solution with tumor lysate (containing $1.5 \mathrm{mg}$ of extract tumor lysate proteins, $3.0-\mathrm{ml}$ final volume) to fabricate the MN matrix. For the blank MNs without tumor lysate, mixed methacrylated hyaluronic acid solution was used without the tumor lysate. For the melanin-loaded MNs, $50-\mu \mathrm{g}$ melanin was dissolved in 4.0 wt \% mixed methacrylated hyaluronic acid solution to fabricate the $\mathrm{MN}$ matrix. The stock melanin solution $(100 \mathrm{mg} / \mathrm{ml})$ was prepared by dissolving melanin in $1.0 \mathrm{M}$ sodium hydroxide and heating to $99^{\circ} \mathrm{C}$ for $10 \mathrm{~min}$. Final formulation was dried at $25^{\circ} \mathrm{C}$ in a vacuum desiccator overnight. After desiccation was completed, needle arrays were carefully separated from the silicone molds and cross-linked via ultraviolet irradiation. The needle base was tailored into a square shape. Fluorescent MNs were fabricated with phalloidin-labeled tumor lysate and rhodamine B- or Cy5.5-labeled hyaluronic acid. The preparation and storage of MNs were under sterilized condition. Morphology of MNs was characterized on a FEI Verios 460L field-emission scanning electron microscope operating at $20 \mathrm{kV}$ after sputter coating with gold/ palladium at the Analytical Instrumentation Facility. Cross sections of MNs were obtained by cutting $5-\mu \mathrm{m}$ slides using Thermo Fisher Scientific HM525 NX Cryostat and stained with Alexa Fluor 488 phalloidin and Hoechst. Fluorescence images of MNs were taken by the Olympus IX70 multiparameter fluorescence microscope.

\section{NIR-responsive property of $\mathbf{M N}$}

To evaluate the property of the MN patch in response to NIR light irradiation, we irradiated samples with 808-nm NIR laser light. The diode infrared laser module (Opto Engine LLC, MDL-N-808) was approved by a laser safety officer of the North Carolina State University (NC State) Environmental Health and Safety Center. The MN patches were placed on a piece of white paper with the needle tips facing down. The laser wire was fixed in place using a plate stand with a support rod and a clamp. The distance between the patch and spotlight was fixed at $10 \mathrm{~cm}$, and the spot size was $\sim 1 \mathrm{~cm}^{2}$. The output energy of the diode infrared laser module was adjusted within $1.0 \mathrm{~W}$ and led to the intensity per unit area $\left(E=I / d^{2}\right)$ of $1.0 \mathrm{~W} / \mathrm{cm}^{2}$ (at $808 \mathrm{~nm}$ ). The NIR light was irradiated continuously or intermittently as needed. Surface temperature changes of the $\mathrm{MN}$ patch backing were recorded, and the highest temperature was controlled below $42^{\circ} \mathrm{C}$ in real time using a thermal imager (FLIRE4) and a noncontact infrared thermometer gun (Leegoal). For intermittent irradiation, the MNs were repeatedly exposed to the laser $\left(1.0 \mathrm{~W} / \mathrm{cm}^{2}\right)$, and the local temperature reached about $42^{\circ} \mathrm{C}$ for $1 \mathrm{~min}$. Subsequently, the laser was turned off for $1 \mathrm{~min}$. This cycle was repeated four times to assess the responsive behavior of MN patch under repeated NIR irradiation. For the continuous irradiation, samples with different parameters were tested. The effect of melanin content on the lightresponsive behavior was tested on MNs loaded with tumor lysate of different protein concentration or blank MN with hyaluronic acid. Quantitative surface temperature changes of the representative MNs were recorded under continuous NIR irradiation at $1.0 \mathrm{~W} / \mathrm{cm}^{2}$. In a separate experiment, the laser power flux was controlled. During continuous NIR exposure for $2 \mathrm{~min}$, the maximal temperature of the MN surface was recorded and plotted. The effect of MN backing thickness on the light-responsive behavior was tested on MNs with continuous NIR irradiation at $1.0 \mathrm{~W} / \mathrm{cm}^{2}$. The thickness of the $\mathrm{MN}$ patch was measured with an average value of $181 \mu \mathrm{m}$ and an SD of $12.5 \mu \mathrm{m}$ using a digimatic indicator (Mitutoyo Corp. ID-C112E Series 543). MN patch samples with different backing thicknesses $(169,175,179,181$, and $202 \mu \mathrm{m})$ were used.

\section{In vitro DC activation}

Bone marrow was collected by flushing the femur and tibia with complete RPMI 1640 containing 10\% fetal bovine growth serum. After lysis of red blood cells (lysate solution, Cwbiotech), $1 \times 10^{6}$ bone marrow cells were seeded in six-well culture dishes with $3.0 \mathrm{ml}$ of the culture medium containing GM-CSF $(20 \mathrm{ng} / \mathrm{ml})$ and $50 \mu \mathrm{M}$ $\beta$-mercaptoethanol (Bio-Rad, Hercules, CA, USA). On day 3 , an additional $4.0 \mathrm{ml}$ of the same medium with GM-CSF was added into the plates. On day 6 , half of the culture supernatant was collected and centrifuged. Cells were resuspended in RPMI 1640 and added back into the original plates. On day 7, nonadherent cells were collected and used as bone marrow-derived DCs for further research use. Another murine DC line JAWS II cells were cultured in minimum essential medium Eagle alpha modification (Sigma-Aldrich) supplemented with GM-CSF $(5 \mathrm{ng} / \mathrm{ml})$. DCs were left unstimulated or stimulated for 12 hours with blank MN (100 $\mu \mathrm{g} / \mathrm{ml})$ or tumor lysate-loaded MN release medium or lipopolysaccharide (LPS; $100 \mathrm{ng} / \mathrm{ml}$ ). After the stimulation, the cells were exposed to NIR irradiation $\left(1.0 \mathrm{~W} / \mathrm{cm}^{2}\right)$ at a distance of $10 \mathrm{~cm}$ for $0,5,10,15$, and $20 \mathrm{~min}$, respectively. After cell incubation for 4 hours, the supernatants were collected and measured by IL-12 p70 mouse ELISA kit (Thermo Fisher Scientific, MC0121). The cells were washed and stained with LIVE/DEAD assay (Thermo Fisher Scientific, L3224) and $\mathrm{CD}^{+} 0^{+}$, and $\mathrm{CD}^{+} 6^{+}$maturation marker-specific antibodies and subsequently analyzed by the confocal microscopy and flow cytometer. Both bone marrow-derived DCs and JAWS II cell line were used for characterization of DC activation. The LIVE/ DEAD assay imaging data were obtained from the JAWS II cell line. 


\section{Mice and in vivo tumor models}

Female C57BL/6J mice, BALB/cJ mice, CD11c-DTR transgenic mice [B6.FVB-Tg(Itgax-DTR/EGFP)57Lan/J; stock no. 004509], and $\mathrm{Rag1}^{-/-}$knockout mice (B6.129S7-Rag1 $1^{\text {tm1Mom }} / \mathrm{J}$; stock no. 002216) were purchased from the Jackson Laboratory. Mice were weighed and randomly divided into different groups. On day 0 , healthy mice were treated with MNs loaded with tumor lysates and GM-CSF, blank MNs loaded with synthetic melanin without tumor lysates, or blank MNs containing hyaluronic acid only (blank). MN patches were applied into the skin of the caudal-dorsal area for about $10 \mathrm{~min}$ and further fixed using Skin Affix surgical adhesive (Medline Industries Inc.). After injection of the MNs, NIR irradiation was performed on the localized $\mathrm{MN}$ region for $10 \mathrm{~min}$ each day for five successive days after immunization (MN + NIR). The diode infrared laser module at $808 \mathrm{~nm}$ (Opto Engine LLC, MDL-N-808) was approved by a laser safety officer of the NC State University Environmental Health and Safety Center. Mice in the control groups were treated with either vaccine MNs without NIR irradiation (MN), blank MNs loaded with synthetic melanin without tumor lysate with NIR irradiation (melanin), or blank MNs containing hyaluronic acid only with NIR irradiation (blank). Surface temperature changes of the regional skin were recorded and controlled below $42^{\circ} \mathrm{C}$ in real time using a thermal imager (FLIR E4) and a noncontact infrared thermometer gun (Leegoal). On day 10,1 × 106 B16F10 tumor cell lines in $25 \mu \mathrm{l}$ of phosphate-buffered saline (PBS) were subcutaneously transplanted into the flank of the C57BL/6J mice, CD11c-DTR transgenic mice, and Rag1 ${ }^{-/-}$knockout mice. Tumor-free mice were rechallenged with $1 \times 10^{6} \mathrm{~B} 16 \mathrm{~F} 10$ tumor cells in $25 \mu \mathrm{l}$ of PBS 80 days after the first tumor inoculation. For the depletion antibody study, we depleted specific T cell, B cell, and NK cell populations in mouse models. Mice were intraperitoneally given $200 \mu \mathrm{g}$ of antibody purified from mouse thymus or spleen dissolved in $200 \mu \mathrm{l}$ of PBS. Antibodies against CD4 (BioLegend, LEAF 100435), CD8 (BioLegend, LEAF 100735), CD19 (BioLegend, LEAF 152402), and NK-1.1 (BioLegend, LEAF 108712) were administered twice weekly for 3 weeks, starting 1 week before the tumor inoculation. Depletions were confirmed by flow cytometry of splenic suspension. For another melanoma model, $1 \times 10^{6} \mathrm{BP}$ tumor cells in $25 \mu$ of PBS were subcutaneously transplanted into the flank of the C57BL/6J mice. For the carcinoma tumor model, $1 \times 10^{6} 4 \mathrm{~T} 1 \mathrm{tu}-$ mor cells in $25 \mu$ l of PBS were subcutaneously transplanted into the flank of the BALB/cJ mice. For experimental metastasis model, $1 \times 10^{5}$ tumor cells were intravenously infused into mice via the tail vein. In another set of experiments, tumor cells were subcutaneously transplanted into the flank of the mice on day 0 . The tumor-bearing mice were weighed and randomly divided into four groups when the tumor volume reached around $50 \mathrm{~mm}^{3}$ on day 3 . After that, the mice were peritumorally administrated with sterile $\mathrm{MN}$ loaded with tumor lysate and GM-CSF (MN) or blank MN containing hyaluronic acid only (blank). After that, NIR light was irradiated on the MN patch for $10 \mathrm{~min}$ during the following 5 days from days 3 to 7 . Tumor growth was measured by a digital caliper or monitored by bioluminescence signals of luciferase-tagged cells. The tumor volume (cubic millimeter) was calculated as $1 / 2 \times$ long diameter $\times(\text { short diameter })^{2}$.

To assess potential toxicity, we monitored mice daily for weight loss. $\mathrm{H} \& \mathrm{E}$ staining was performed on the organs collected from the mice following the standard procedure (Histology Laboratory at NC State College of Veterinary Medicine). Lungs were excised, and macroscopically visible metastases were counted. The resected primary tumors were stored at $-20^{\circ} \mathrm{C}$ for immunofluorescence staining or fixed in $4 \%$ paraformaldehyde for subsequent analysis.

\section{Identification of DC subsets, T cells, and cytokines}

Antibodies used were purchased from Thermo Fisher Scientific and BioLegend Inc. Staining antibodies, including CD3 (Thermo Fisher Scientific, A18644), CD4 (Thermo Fisher Scientific, A18667), CD8 (Thermo Fisher Scientific, A18609), CD11c(BioLegend, 117309), CD49b (BioLegend, 108909), CD80 (BioLegend, 104707), CD86 (BioLegend, 105007), PIR-A/B (gp91, BioLegend, 144103), tetramer (MBL International, H-2D ${ }^{\mathrm{b}}$ gp100, EGSRNQDWL-P), and fluorogenic CellROX Deep Red reagent (BioLegend, C10422) were used for fluorescenceactivated cell sorting (FACS) and were analyzed following the manufacturers' instructions. Organs (skin, tumor, lymph node, and spleen) were harvested and minced into 2- to 4-mm pieces using scissors or scalpel blade. A single-cell suspension was prepared in the cell staining buffer (BioLegend, 420201). CellROX reagents at predetermined optimum concentrations were added to the cells and incubated on ice for 20 to $30 \mathrm{~min}$ in the dark for ROS detection. The relevant tetramer phycoerythrin (PE) was stained at room temperature for $30 \mathrm{~min}$ in the dark before additional staining with appropriately conjugated fluorescent antibodies. Stained cells were analyzed on a FACSCalibur instrument (BD) and using FlowJo software. To determine the concentration of different cytokines at the vaccine MN site, patch and adjacent tissues were excised and digested with T-PER (Pierce). Cytokine concentrations in the extracted patch were analyzed with a BioLegend's LEGENDplex beadbased immunoassays, according to the manufacturer's instructions.

\section{Statistical analysis}

Statistical analysis was evaluated using GraphPad Prism (6.0). Statistical analysis was performed with paired Student's $t$ test and analysis of variance (ANOVA). $P$ values for Kaplan-Meier curves were calculated with log-rank test. $P$ values of 0.05 or less were considered significant.

\section{Study approval}

All mouse studies were performed following animal protocols approved by the Institutional Animal Care and Use Committee at the NC State University and the University of North Carolina at Chapel Hill (UNC-CH).

\section{SUPPLEMENTARY MATERIALS}

immunology.sciencemag.org/cgi/content/full/2/17/eaan5692/DC1 Materials and Methods

Fig. S1. Mechanical property of the MN.

Fig. S2. Characteristics of tumor lysate solution and synthetic melanin.

Fig. S3. Heating behavior of MN patches by repetitive NIR irradiation.

Fig. S4. Surface temperature of MN patches with various loadings of tumor lysates upon NIR irradiation.

Fig. S5. In vitro release profiles of GM-CSF and tumor lysate proteins.

Fig. S6. Scanning electron microscopy images of MN patch after insertion into the mouse skin over time.

Fig. S7. DC function evaluation after in vitro activation.

Fig. S8. Cytotoxicity study of the blank MNs.

Fig. S9. Characterization of the skin after MN insertion.

Fig. S10. Melanin-loaded MNs confer protective immunity in vivo.

Fig. S11. Tumor growth in control and treated mice.

Fig. S12. Quantified B16F10 bioluminescent tumor signals in control and treated mice.

Fig. S13. Tumor weights in control and treated mice.

Fig. S14. Histology and apoptosis analysis of the tumor sections.

Fig. S15. Tumor growth of mice receiving the transdermal cancer immunotherapy.

Fig. S16. Measurement of local microcirculatory blood perfusion of mice.

Fig. S17. Immunologic responses after the transdermal cancer immunotherapy.

Fig. S18. Quantification of IgG1 subtypes in serum after treatment with blank, MN, or MN + NIR.

Fig. S19. ROS detection by flow cytometry in tumor sections.

Fig. S20. HSP90 expression after the transdermal cancer immunotherapy.

Fig. S21. Cytokine kinetics after the transdermal cancer immunotherapy. 
Fig. S22. Histology analysis after the transdermal cancer immunotherapy.

Fig. S23. Antitumor effect of the transdermal cancer immunotherapy toward different tumor models.

Fig. S24. Surface temperature changes of the melanin-loaded MNs.

Fig. S25. Antitumor effect of the transdermal cancer immunotherapy.

Fig. S26. HSP70 expression after the transdermal cancer immunotherapy.

Fig. S27. Representative quantitative analysis of the DC activation.

Fig. S28. Cytokine kinetics after the transdermal cancer immunotherapy.

Fig. S29. Average weights of mice after the transdermal cancer immunotherapy in control and treated mice.

Fig. S30. H\&E staining of organs collected after the transdermal cancer immunotherapy. Table S1. Melanin content of tumors excised from tumor-bearing mice.

Table S2. Measurement of total local microcirculatory blood perfusion of mice receiving different treatments using the laser Doppler flowmetry.

Excel file 1.

Excel file 2 .

\section{REFERENCES AND NOTES}

1. J. Couzin-Frankel, Cancer immunotherapy. Science 342, 1432-1433 (2013).

2. D. Ricklin, G. Hajishengallis, K. Yang, J. D. Lambris, Complement: A key system for immune surveillance and homeostasis. Nat. Immunol. 11, 785-797 (2010).

3. J. B. Swann, M. J. Smyth, Immune surveillance of tumors. J. Clin. Invest. 117, 1137-1146 (2007).

4. L. Gu, D. J. Mooney, Biomaterials and emerging anticancer therapeutics: Engineering the microenvironment. Nat. Rev. Cancer 16, 56-66 (2015).

5. L. E. Klevorn, R. M. Teague, Adapting cancer immunotherapy models for the real world. Trends Immunol. 37, 354-363 (2016).

6. D. R. Littman, Releasing the brakes on cancer immunotherapy. Cell 162, 1186-1190 (2015).

7. C. G. Drake, E. J. Lipson, J. R. Brahmer, Breathing new life into immunotherapy: Review of melanoma, lung and kidney cancer. Nat. Rev. Clin. Oncol. 11, 24-37 (2013).

8. M. E. Dudley, S. A. Rosenberg, Adoptive-cell-transfer therapy for the treatment of patients with cancer. Nat. Rev. Cancer 3, 666-675 (2003).

9. S. A. Rosenberg, N. P. Restifo, J. C. Yang, R. A. Morgan, M. E. Dudley, Adoptive cell transfer: A clinical path to effective cancer immunotherapy. Nat. Rev. Cancer 8, 299-308 (2008).

10. M. T. Stephan, J. J. Moon, S. H. Um, A. Bershteyn, D. J. Irvine, Therapeutic cell engineering with surface-conjugated synthetic nanoparticles. Nat. Med. 16, 1035-1041 (2010).

11. B. Huang, W. D. Abraham, Y. Zheng, S. C. Bustamante López, S. S. Luo, D. J. Irvine, Active targeting of chemotherapy to disseminated tumors using nanoparticle-carrying $T$ cells. Sci. Transl. Med. 7, 291 ra94 (2015)

12. D. J. Irvine, M. A. Swartz, G. L. Szeto, Engineering synthetic vaccines using cues from natural immunity. Nat. Mater. 12, 978-990 (2013)

13. M. F. Bachmann, G. T. Jennings, Vaccine delivery: A matter of size, geometry, kinetics and molecular patterns. Nat. Rev. Immunol. 10, 787-796 (2010).

14. J. Banchereau, A. K. Palucka, Dendritic cells as therapeutic vaccines against cancer. Nat. Rev. Immunol. 5, 296-306 (2005).

15. M. V. Dhodapkar, M. Sznol, B. Zhao, D. Wang, R. D. Carvajal, M. L. Keohan, E. Chuang, R. E. Sanborn, J. Lutzky, J. Powderly, H. Kluger, S. Tejwani, J. Green, V. Ramakrishna, A. Crocker, L. Vitale, M. Yellin, T. Davis, T. Keler, Induction of antigen-specific immunity with a vaccine targeting NY-ESO-1 to the dendritic cell receptor DEC-205. Sci. Transl. Med. 6, 232ra51 (2014).

16. A. D. Garg, L. Vandenberk, C. Koks, T. Verschuere, L. Boon, S. W. Van Gool, P. Agostinis, Dendritic cell vaccines based on immunogenic cell death elicit danger signals and T cell-driven rejection of high-grade glioma. Sci. Transl. Med. 8, 328ra27 (2016).

17. T. R. Fadel, F. A. Sharp, N. Vudattu, R. Ragheb, J. Garyu, D. Kim, E. P. Hong, N. Li, G. L. Haller, L. D. Pfefferle, S. Justesen, K. C. Herold, T. M. Fahmy, Corrigendum: A carbon nanotube-polymer composite for T-cell therapy. Nat. Nanotechnol. 9, 723 (2014).

18. J. V. Kim, J.-B. Latouche, I. Rivière, M. Sadelain, The ABCs of artificial antigen presentation. Nat. Biotechnol. 22, 403-410 (2004).

19. C. L.-L. Chiang, G. Coukos, L. Kandalaft, Whole tumor antigen vaccines: Where are we? Vaccines 3, 344-372 (2015).

20. W. Kratky, C. Reis e Sousa, A. Oxenius, R. Spörri, Direct activation of antigen-presenting cells is required for $\mathrm{CD}^{+}{ }^{+}$T-cell priming and tumor vaccination. Proc. Natl. Acad. Sci. U.S.A. 108, 17414-17419 (2011).

21. S. B. Stephan, A. M. Taber, I. Jileaeva, E. P. Pegues, C. L. Sentman, M. T. Stephan, Biopolymer implants enhance the efficacy of adoptive T-cell therapy. Nat. Biotechnol. 33, 97-101 (2015).

22. J. Kim, W. A. Li, Y. Choi, S. A. Lewin, C. S. Verbeke, G. Dranoff, D. J. Mooney, Injectable, spontaneously assembling, inorganic scaffolds modulate immune cells in vivo and increase vaccine efficacy. Nat. Biotechnol. 33, 64-72 (2015).
23. O. A. Ali, D. Emerich, G. Dranoff, D. J. Mooney, In situ regulation of DC subsets and T cells mediates tumor regression in mice. Sci. Transl. Med. 1, 8 ra19 (2009).

24. S. S. Evans, E. A. Repasky, D. T. Fisher, Fever and the thermal regulation of immunity: The immune system feels the heat. Nat. Rev. Immunol. 15, 335-349 (2015).

25. K. Sato, N. Sato, B. Xu, Y. Nakamura, T. Nagaya, P. L. Choyke, Y. Hasegawa, H. Kobayashi, Spatially selective depletion of tumor-associated regulatory $\mathrm{T}$ cells with near-infrared photoimmunotherapy. Sci. Transl. Med. 8, 352ra110 (2016).

26. J. Fu, D. B. Kanne, M. Leong, L. H. Glickman, S. M. McWhirter, E. Lemmens, K. Mechette, J. J. Leong, P. Lauer, W. Liu, K. E. Sivick, Q. Zeng, K. C. Soares, L. Zheng, D. A. Portnoy, J. J. Woodward, D. M. Pardoll, T. W. Dubensky Jr., Y. Kim, STING agonist formulated cancer vaccines can cure established tumors resistant to PD-1 blockade. Sci. Transl. Med. 7, 283ra52 (2015).

27. T. S. Kupper, R. C. Fuhlbrigge, Immune surveillance in the skin: Mechanisms and clinical consequences. Nat. Rev. Immunol. 4, 211-222 (2004).

28. K. Nagao, F. Ginhoux, W. W. Leitner, S.-I. Motegi, C. L. Bennett, B. E. Clausen, M. Merad, M. C. Udey, Murine epidermal Langerhans cells and langerin-expressing dermal dendritic cells are unrelated and exhibit distinct functions. Proc. Natl. Acad. Sci. U.S.A. 106, 3312-3317 (2009).

29. C. Wang, Y. Ye, G. M. Hochu, H. Sadeghifar, Z. Gu, Enhanced cancer immunotherapy by microneedle patch-assisted delivery of anti-PD1 antibody. Nano Lett. 16, 2334-2340 (2016).

30. J. Yu, Y. Zhang, Y. Ye, R. DiSanto, W. Sun, D. Ranson, F. S. Ligler, J. B. Buse, Z. Gu, Microneedle-array patches loaded with hypoxia-sensitive vesicles provide fast glucose-responsive insulin delivery. Proc. Natl. Acad. Sci. U.S.A. 112, 8260-8265 (2015).

31. A. Filson, J. Hope, Isolation of melanin granules. Nature 179, 211 (1957).

32. A. J. Lea, Solubility of melanins. Nature 170, 709 (1952).

33. R. Zhang, Q. Fan, M. Yang, K. Cheng, X. Lu, L. Zhang, W. Huang, Z. Cheng, Engineering melanin nanoparticles as an efficient drug-delivery system for imaging-guided chemotherapy. Adv. Mater. 27, 5063-5069 (2015).

34. S. P. Sullivan, D. G. Koutsonanos, M. del Pilar Martin, J. W. Lee, V. Zarnitsyn, S.-O. Choi, N. Murthy, R. W. Compans, I. Skountzou, M. R. Prausnitz, Dissolving polymer microneedle patches for influenza vaccination. Nat. Med. 16, 915-920 (2010).

35. M. L. Moraes, P. J. Gomes, P. A. Ribeiro, P. Vieira, A. A. Freitas, R. Kohler, O. N. Oliveira Jr., M. Raposo, Polymeric scaffolds for enhanced stability of melanin incorporated in liposomes. J. Colloid Interface Sci. 350, 268-274 (2010).

36. B. M. Carreno, V. Magrini, M. Becker-Hapak, S. Kaabinejadian, J. Hundal, A. A. Petti, A. Ly, W. R. Lie, W. H. Hildebrand, E. R. Mardis, G. P. Linette, A dendritic cell vaccine increases the breadth and diversity of melanoma neoantigen-specific T cells. Science $\mathbf{3 4 8}, 803-808$ (2015).

37. J. C. Castle, S. Kreiter, J. Diekmann, M. Löwer, N. van de Roemer, J. de Graaf, A. Selmi, M. Diken, S. Boegel, C. Paret, M. Koslowski, A. N. Kuhn, C. M. Britten, C. Huber, Ö. Türeci, U. Sahin, Exploiting the mutanome for tumor vaccination. Cancer Res. 72, 1081-1091 (2012).

38. A. Murshid, J. Gong, S. K. Calderwood, The role of heat shock proteins in antigen cross presentation. Front. Immunol. 3, 63 (2012).

39. M. M. Hoffmann, C. Molina-Mendiola, A. D. Nelson, C. A. Parks, E. E. Reyes, M. J. Hansen, G. Rajagopalan, L. R. Pease, A. G. Schrum, D. Gil, Co-potentiation of antigen recognition: A mechanism to boost weak T cell responses and provide immunotherapy in vivo. Sci. Adv. 1, e1500415 (2015).

40. D. Dankort, D. P. Curley, R. A. Cartlidge, B. Nelson, A. N. Karnezis, W. E. Damsky Jr., M. J. You, R. A. DePinho, M. McMahon, M. Bosenberg, Braf ${ }^{\mathrm{V} 600 \mathrm{E}}$ cooperates with Pten loss to induce metastatic melanoma. Nat. Genet. 41, 544-552 (2009).

41. A. Holtzhausen, F. Zhao, K. S. Evans, M. Tsutsui, C. Orabona, D. S. Tyler, B. A. Hanks, Melanoma-derived Wnt5a promotes local dendritic-cell expression of IDO and immunotolerance: Opportunities for pharmacologic enhancement of immunotherapy. Cancer Immunol. Res. 3, 1082-1095 (2015).

42. Q. Chen, L. Xu, C. Liang, C. Wang, R. Peng, Z. Liu, Photothermal therapy with immune-adjuvant nanoparticles together with checkpoint blockade for effective cancer immunotherapy. Nat. Commun. 7, 13193 (2016).

43. J. Conde, N. Oliva, Y. Zhang, N. Artzi, Local triple-combination therapy results in tumour regression and prevents recurrence in a colon cancer model. Nat. Mater. 15, 1128-1138 (2016).

44. W. Sun, Q. Hu, W. Ji, G. Wright, Z. Gu, Leveraging physiology for precision drug delivery. Physiol. Rev. 97, 189-225 (2017).

45. Y. Zhang, J. Yu, A. Kahkoska, Z. Gu, Photoacoustic drug delivery. Sensors 17, 1400 (2017).

46. L. Cheng, C. Wang, L. Feng, K. Yang, Z. Liu, Functional nanomaterials for phototherapies of cancer. Chem. Rev. 114, 10869-10939 (2014).

47. C. Wang, Y. Ye, Q. Hu, A. Bellotti, Z. Gu, Tailoring biomaterials for cancer immunotherapy: Emerging trends and future outlook. Adv. Mater. 29, 1606036 (2017).

Acknowledgments: We thank L. Huang at the UNC-CH for providing the B16F10-Luc cell line and $B R A F^{V 600 E}{ }^{2} T_{E N}^{-/-}$Duke-clone 6 cell line (generated in the laboratory of B. A. Hanks at Duke Cancer Institute), B. Andersen at the NC State University for Fourier transform infrared analysis, 
and the UNC Research Opportunity Initiative for the strain tests. Funding: This work was supported by grants from the NC Translational and Clinical Sciences, the NIH Clinical and Translational Science Awards (NIH grant 1L1TR001111) at the UNC-CH, the Sloan Research Fellowship of the Alfred P. Sloan Foundation, and the pilot grant from the UNC Lineberger Comprehensive Cancer Center. Author contributions: Y.Y., C.W., L.H., G.D., and Z.G. designed the experiments. Y.Y., C.W., and X.Z. performed the experiments. Y.Y. and C.W. performed the statistical analyses. Y.Y., C.W., Q.H., Y.Z., Q.L., D.W., J.M., A.B., L.H., G.D., and Z.G. analyzed the data and wrote the paper. Competing interests: The authors declare that they have no competing interests.
Submitted 2 May 2017

Accepted 11 October 2017

Published 10 November 2017

10.1126/sciimmunol.aan5692

Citation: Y. Ye, C. Wang, X. Zhang, Q. Hu, Y. Zhang, Q. Liu, D. Wen, J. Milligan, A. Bellotti, L. Huang, G. Dotti, Z. Gu, A melanin-mediated cancer immunotherapy patch. Sci. Immunol. 2, eaan5692 (2017). 